\title{
GMPCP FOR ONE ROW AND ONE COLUMN COMPLETION*
}

\author{
MARIJA DODIG ${ }^{\dagger}$ AND MARKO STOŠIĆ $\ddagger$
}

\begin{abstract}
In this paper, the possible Kronecker invariants of a matrix pencil obtained by simultaneous one row and one column completion of a given matrix pencil are characterized. This presents a new approach to the General Matrix Pencil Completion Problem (GMPCP), where simultaneous row and column completion is considered.
\end{abstract}

Key words. Kronecker invariants, Completion of matrix pencils, Partitions of integers.

AMS subject classifications. 15A83, 05A20.

1. Introduction. The General Matrix Pencil Completion Problem (GMPCP) consists of characterizing the possible Kronecker invariants of a matrix pencil with a prescribed subpencil. It was posed in [18] as one of the Challenging Problems of Modern Linear Algebra. Apart from its theoretical importance, it has many applications in Control Theory, Graph Theory, Representation Theory of quivers, etc. (see e.g. [18] and references therein).

There are two main approaches in attacking GMPCP: minimal and classical one. The advantage of the minimal one is that it has no restrictions on the structure of the involved pencils, only on their dimensions. On the other hand, the power of the classical approach is that by restricting the involved invariants, we can develop techniques and methods that will work on arbitrary pencils too, thus developing a path toward general solution. In this paper, we join the two approaches and resolve GMPCP for one row and one column completion.

We have been studying GMPCP for years, and many partial results have been obtained. For example, by using the minimal approach GMPCP has been fully resolved in [3]. However in the minimal approach we have certain restrictions on the possible dimension and rank of the resulting pencil. Therefore, GMPCP is still open in full generality. On the other hand, following the classical approach in $[6,12]$ we have solved GMPCP only for row, or equivalently column, completions, but not simultaneously. For general completions, in $[2,4]$, GMPCP has been solved for the case when either resulting or prescribed pencil is regular. In $[7,8]$, GMPCP has been solved for the case when either resulting or prescribed pencil has column minimal indices as the only nontrivial Kronecker invariants. In [5,9], GMPCP has been solved when both of the involved pencils are quasi-regular. Finally, we made recent breakthrough in $[10,11]$ and solved GMPCP for the case when only one of the involved pencils (resulting or prescribed one) is quasi-regular. In all these papers we need restrictions on the structure of the involved pencils, but none is imposed on their sizes or ranks.

*Received by the editors on January 29, 2020. Accepted for publication on May 31, 2020. Handling Editor: João Filipe Queiró. Corresponding Author: Marija Dodig. This work was done within the activities of CEAFEL and was partially supported by FCT projects UIDB/04721/2020, Exploratory Grants IF/01232/2014/CP1216/CT0012 (M.D.) and IF/0998/2015 (M.S.), and by the Ministry of Education, Science and Technological Development of Republic of Serbia, projects no. 174020 (M.D) and no. 174012 (M.S.)

${ }^{\dagger}$ CEAFEL, Departamento de Matématica, Universidade de Lisboa, Edificio C6, Campo Grande, 1749-016 Lisbon, Portugal (msdodig@fc.ul.pt), and Mathematical Institute SANU, Knez Mihajlova 36, 11000 Beograd, Serbia.

${ }^{\ddagger}$ CAMGSD, Departamento de Matemática, Instituto Superior Técnico, Av. Rovisco Pais 1, 1049-001 Lisbon, Portugal, and Mathematical Institute SANU, Knez Mihajlova 36, 11000 Belgrade, Serbia. 
In this paper, we deal with arbitrary pencils, so there are no structural restrictions. That makes the problem very "wild". Since GMPCP is still open and the existing methods are still missing the final touch, in this paper, we solve it for one row and one column case. It is the first non-trivial case significantly different from the existing ones, and in the future it could be used as a base of the induction for solving GMPCP.

In this paper, we study and solve the following problem:

Problem 1. Let $A(\lambda) \in \mathbb{F}[\lambda]^{(n+p) \times(n+m)}$ and $M(\lambda) \in \mathbb{F}[\lambda]^{(n+p+1) \times(n+m+1)}$ be matrix pencils. Find necessary and sufficient conditions for the existence of pencils $x(\lambda) \in \mathbb{F}[\lambda]^{(n+p) \times 1}, y(\lambda) \in \mathbb{F}[\lambda]^{1 \times(n+m)}$, and $z(\lambda) \in \mathbb{F}[\lambda]^{1 \times 1}$, such that the pencil

$$
\left[\begin{array}{c|c}
A(\lambda) & x(\lambda) \\
\hline y(\lambda) & z(\lambda)
\end{array}\right]
$$

is strictly equivalent to $M(\lambda)$.

Strict equivalence of matrix pencils will be quickly reviewed in Section 2.2.

Let

$$
l:=\operatorname{rank} M(\lambda)-\operatorname{rank} A(\lambda) .
$$

An obvious necessary condition for Problem 1 is that

$$
0 \leq l \leq 2 .
$$

Depending on the value of $l$, we shall consider and resolve three problems. In fact, for $l=0$ and $l=2$, Problem 1 belongs to the so called minimal problems, and thus, it is already resolved by [3, Theorems 4.1 and 4.2]. So the only new result comes from the case $l=1$. In Section 3 we solve Problem 1 for the case $l=1$, and we cite results that solve Problem 1 in the remaining cases $(l=0$ and $l=2)$. We hope that the main result of the paper, Theorem 3.1 will help in better understanding and solving GMPCP.

2. Notation and auxiliary results. Let $\mathbb{F}$ be a field. All polynomials in the paper are considered to be monic and homogeneous. For details on homogeneous polynomials, see e.g. [16] and [17, Chapter 2]. Let $\alpha_{1}|\cdots| \alpha_{n}$ be homogeneous invariant factors of a matrix pencil $A(\lambda)$. We assume that $\alpha_{i}=1$ for all $i<1$, and $\alpha_{i}=0$ for all $i>n$. By $d\left(\alpha_{i}\right)$ we denote the degree of a polynomial $\alpha_{i}$.

2.1. Partitions and generalized majorization. By a partition we mean a non increasing sequence of integers. For any integers $a_{1} \geq \cdots \geq a_{s}$, we denote a corresponding partition by $\mathbf{a}=\left(a_{1}, \ldots, a_{s}\right)$. Moreover, for any such sequence, we assume that $a_{i}=+\infty$ for $i \leq 0$, and $a_{i}=-\infty$ for $i>s$. Also, for any integers $c_{1}, \ldots, c_{r}$, we put $\sum_{i=a}^{b} c_{i}=0$ whenever $a>b$.

We shall use the concept of generalized majorizations between three partitions (for details, see e.g. [8]):

Definition 2.1. Let $\mathbf{d}=\left(d_{1}, \ldots, d_{m}\right), \mathbf{g}=\left(g_{1}, \ldots, g_{m+s}\right)$ and $\mathbf{a}=\left(a_{1}, \ldots, a_{s}\right)$ be partitions. If

$$
\begin{gathered}
d_{i} \geq g_{i+s}, \quad i=1, \ldots, m, \\
\sum_{i=1}^{h_{j}} g_{i}-\sum_{i=1}^{h_{j}-j} d_{i} \leq \sum_{i=1}^{j} a_{i}, \quad j=1, \ldots, s, \\
\sum_{i=1}^{m+s} g_{i}=\sum_{i=1}^{m} d_{i}+\sum_{i=1}^{s} a_{i},
\end{gathered}
$$


where

$$
h_{j}:=\min \left\{i \mid d_{i-j+1}<g_{i}\right\}, \quad j=1, \ldots, s,
$$

then we say that $\mathbf{g}$ is majorized by $\mathbf{d}$ and $\mathbf{a}$. This type of majorization we call the generalized majorization, and we write

$$
\mathbf{g} \prec^{\prime}(\mathbf{d}, \mathbf{a}) .
$$

In the solution for the case $l=1$, we shall use brute force and some combinatorics from [13], see also [1, Lemma 5.4]:

Theorem 2.2. ([13, Theorem 2.4]) Let $\mathbf{c}=\left(c_{1}, \ldots, c_{m}\right)$ and $\mathbf{d}=\left(d_{1}, \ldots, d_{m}\right)$ be partitions of nonnegative integers. Let $a, b$ be integers. Let $w=\max \left\{i \in\{1, \ldots, m\} \mid c_{i} \neq d_{i}\right\}$, and let $w=0$ if $c_{i}=d_{i}$, for all $i=1, \ldots, m$. If $w>0$, without loss of generality, we assume that $c_{w}>d_{w}$.

There exists a partition $\mathbf{g}=\left(g_{1}, \ldots, g_{m+1}\right)$ of nonnegative integers, such that

$$
\mathbf{g} \prec^{\prime}(\mathbf{d}, a) \quad \text { and } \mathbf{g} \prec^{\prime}(\mathbf{c}, b)
$$

if and only if

$$
\begin{aligned}
& \sum_{i=1}^{m} c_{i}+b=\sum_{i=1}^{m} d_{i}+a \geq 0 \\
& \sum_{i=1}^{m} \min \left(c_{i}, d_{i}\right)+\max \left(c_{h}, d_{h}\right) \geq \sum_{i=1}^{m} d_{i}+a
\end{aligned}
$$

where $h=\max \left\{i \in\{1, \ldots, w\} \mid c_{i}<d_{i-1}\right\}$, and $h=0$ if $w=0$.

REMARK 2.3. In applications of the result from Theorem 2.2, we cannot always assume that $c_{w}>d_{w}$. In order to fix this, Theorem 2.2 is valid without that assumption if we slightly change the definition of $h$. If we define $h$ in the following way:

$$
h:= \begin{cases}\max \left\{i \in\{1, \ldots, w\} \mid c_{i}<d_{i-1}\right\}, & \text { if } c_{w}>d_{w} \\ \max \left\{i \in\{1, \ldots, w\} \mid d_{i}<c_{i-1}\right\}, & \text { if } d_{w}>c_{w} \\ 0 & \text { if } w=0\end{cases}
$$

then Theorem 2.2 holds without assuming $c_{w}>d_{w}$.

2.2. Matrix pencils. Let $E(\lambda), E^{\prime}(\lambda) \in \mathbb{F}[\lambda]^{n \times m}$ be matrix pencils. We say that they are strictly equivalent, denoted by $E(\lambda) \sim E^{\prime}(\lambda)$, if and only if there exist invertible matrices $P \in \mathbb{F}^{n \times n}$ and $Q \in \mathbb{F}^{m \times m}$ such that

$$
E^{\prime}(\lambda)=P E(\lambda) Q
$$

The complete set of strict equivalence invariants (also called the Kronecker invariants) of a matrix pencil consists of its invariant factors, infinite elementary divisors, column and row minimal indices. In this paper, we consider invariant factors and infinite elementary divisors of a pencil unified as homogeneous invariant factors; for details, see $[16,17]$.

The number of Kronecker invariants of a pencil can be expressed in terms of the size and the rank of a matrix pencil as follows: a pencil $E(\lambda) \in F[\lambda]^{(n+p) \times(n+m)}$ with $n=\operatorname{rank} E(\lambda)$, has $n$ homogeneous invariant factors, $p$ (the number of rows minus the rank of $E(\lambda)$ ) row minimal indices, $m$ (the number of columns 
minus the rank of $E(\lambda))$ column minimal indices. Also, the sum of the column minimal indices, the row minimal indices and the degrees of the homogeneous invariant factors of $E(\lambda)$ equals its rank $(n)$. For more details on Kronecker invariants and Kronecker canonical form, see chapter XII of [14].

In the proof of the main result, we shall use [7, Theorem 2] for the case of one row completion. We cite it here in the form from [13]:

Theorem 2.4. ([13, Theorem 2.2], [7, Theorem 2]) Let $C(\lambda) \in \mathbb{F}[\lambda]^{(n+p) \times(n+m)}, \operatorname{rank} C(\lambda)=n$, be a matrix pencil with $\gamma_{1}|\cdots| \gamma_{n}, d_{1} \geq \cdots \geq d_{m}$ and $\bar{r}_{1} \geq \cdots \geq \bar{r}_{p}$ as homogeneous invariant factors, column and row minimal indices, respectively.

Let $A(\lambda) \in \mathbb{F}[\lambda]^{(n+p-1) \times(n+m)}$, $\operatorname{rank} A(\lambda)=n-q$, be a matrix pencil with $\alpha_{1}|\cdots| \alpha_{n-q}, c_{1} \geq \cdots \geq c_{m+q}$ and $r_{1} \geq \cdots \geq r_{p-1+q}$ as homogeneous invariant factors, column and row minimal indices, respectively. Hence, $q=\operatorname{rank} C(\lambda)-\operatorname{rank} A(\lambda)$.

There exists a pencil $y(\lambda) \in \mathbb{F}[\lambda]^{1 \times(n+m)}$ such that

$$
\left[\frac{A(\lambda)}{y(\lambda)}\right]
$$

is strictly equivalent to $C(\lambda)$ if and only if the following conditions are satisfied:

(i) $0 \leq q \leq 1$

(ii) $\gamma_{i}\left|\alpha_{i}\right| \gamma_{i+1}, \quad i=1, \ldots, n-q$,

(iii) if $q=1$, then

$$
\begin{aligned}
& \bar{r}_{i}=r_{i}, \quad i=1, \ldots, p, \text { and } \mathbf{c} \prec^{\prime}(\mathbf{d}, a), \text { with } a=\sum_{i=1}^{m+1} c_{i}-\sum_{i=1}^{m} d_{i} \text {; } \\
& \text { if } q=0 \text {, then } \\
& d_{i}=c_{i}, \quad i=1, \ldots, m, \text { and } \overline{\mathbf{r}} \prec^{\prime}(\mathbf{r}, b), \text { with } b=\sum_{i=1}^{p} \bar{r}_{i}-\sum_{i=1}^{p-1} r_{i} \text {. }
\end{aligned}
$$

In the following section, we solve Problem 1.

3. A solution to Problem 1. Let us consider the pencils $A(\lambda) \in \mathbb{F}[\lambda]^{(n+p) \times(n+m)}$ and $M(\lambda) \in$ $\mathbb{F}[\lambda]^{(n+p+1) \times(n+m+1)}$ from Problem 1.

Let $n=\operatorname{rank} A(\lambda)$, and let $\alpha_{1}|\cdots| \alpha_{n}$ be homogeneous invariant factors, $c_{1} \geq \cdots \geq c_{m}$ be column minimal indices, and $r_{1} \geq \cdots \geq r_{p}$ be row minimal indices of $A(\lambda)$.

Let $n+l=\operatorname{rank} M(\lambda)$, and let $\gamma_{1}|\cdots| \gamma_{n+l}$ be homogeneous invariant factors, $d_{1} \geq \cdots \geq d_{m+1-l}$ be column minimal indices, and $\bar{r}_{1} \geq \cdots \geq \bar{r}_{p+1-l}$ be row minimal indices of $M(\lambda)$.

By (1.2), we have three possibilities on the value of $l$. Depending on the value of $l$ we split Problem 1 into three cases:

3.1. Case $l=1$. Let $w=\max \left\{i \in\{1, \ldots, m\} \mid c_{i} \neq d_{i}\right\}$, if any such $i$ exists, and let $w=0$ if $c_{i}=d_{i}$, for all $i=1, \ldots, m$. Let $z=\max \left\{i \in\{1, \ldots, p\} \mid r_{i} \neq \bar{r}_{i}\right\}$, if any such $i$ exists, and let $z=0$ if $r_{i}=\bar{r}_{i}$, for all $i=1, \ldots, p$. 
Also, let us define integers $h$ and $h^{\prime}$ in the following way:

$$
\begin{gathered}
h:= \begin{cases}\max \left\{i \in\{1, \ldots, w\} \mid c_{i}<d_{i-1}\right\}, & \text { if } w>0 \text { and } c_{w}>d_{w}, \\
\max \left\{i \in\{1, \ldots, w\} \mid d_{i}<c_{i-1}\right\}, & \text { if } w>0 \text { and } d_{w}>c_{w}, \\
0, & \text { if } w=0,\end{cases} \\
h^{\prime}:= \begin{cases}\max \left\{i \in\{1, \ldots, z\} \mid r_{i}<\bar{r}_{i-1}\right\}, & \text { if } r_{z}>\bar{r}_{z}, \\
\max \left\{i \in\{1, \ldots, z\} \mid \bar{r}_{i}<r_{i-1}\right\}, & \text { if } \bar{r}_{z}>r_{z}, \\
0, & \text { if } z=0 .\end{cases}
\end{gathered}
$$

Since $l=1,(1.1)$ gives:

$$
\sum_{i=1}^{m} c_{i}+\sum_{i=1}^{p} r_{i}+\sum_{i=1}^{n} d\left(\alpha_{i}\right)+1=\sum_{i=1}^{m} d_{i}+\sum_{i=1}^{p} \bar{r}_{i}+\sum_{i=1}^{n+1} d\left(\gamma_{i}\right)
$$

By using the above notation, in the following theorem we give a complete solution to Problem 1 in the case $l=1$. This is the main result of the paper:

Theorem 3.1. (Solution to Problem 1 in Case $l=1$ ) There exist pencils $x(\lambda) \in \mathbb{F}[\lambda]^{(n+p) \times 1}, y(\lambda) \in$ $\mathbb{F}[\lambda]^{1 \times(n+m)}$, and $z(\lambda) \in \mathbb{F}[\lambda]^{1 \times 1}$, such that the pencil

$$
\left[\begin{array}{l|l}
A(\lambda) & x(\lambda) \\
\hline y(\lambda) & z(\lambda)
\end{array}\right]
$$

is strictly equivalent to $M(\lambda)$, if and only the following conditions are valid

(i) $\gamma_{i}\left|\alpha_{i}\right| \gamma_{i+2}, \quad i=1, \ldots, n$,

(ii) one of the following sets of conditions is satisfied

$$
\begin{aligned}
& \text { (I) } \quad c_{i}=d_{i}, \quad i=1, \ldots, m, \text { and } \\
& \sum_{i=1}^{p} \min \left(r_{i}, \bar{r}_{i}\right)+\max \left(r_{h^{\prime}}, \bar{r}_{h^{\prime}}\right) \geq \sum_{i=1}^{p} \bar{r}_{i}+\sum_{i=1}^{n+1} d\left(\gamma_{i}\right)-\sum_{i=1}^{n} d\left(\operatorname{gcd}\left(\alpha_{i}, \gamma_{i+1}\right)\right)-1, \\
& \quad r_{i}=\bar{r}_{i}, \quad i=1, \ldots, p, \quad \text { and } \\
& \sum_{i=1}^{m} \min \left(c_{i}, d_{i}\right)+\max \left(c_{h}, d_{h}\right) \geq \sum_{i=1}^{m} d_{i}+\sum_{i=1}^{n+1} d\left(\gamma_{i}\right)-\sum_{i=1}^{n} d\left(\operatorname{gcd}\left(\alpha_{i}, \gamma_{i+1}\right)\right)-1 .
\end{aligned}
$$

Proof. Let us consider the pencil

$$
[A(\lambda) \mid x(\lambda)] \in \mathbb{F}[\lambda]^{(n+p) \times(n+m+1)}
$$

Then we have that $\operatorname{rank}[A(\lambda) \mid x(\lambda)]=n+s_{1}$, where $s_{1} \in\{0,1\}$. Let us denote homogeneous invariant factors of $(3.11)$ by $\beta_{1}|\cdots| \beta_{n+s_{1}}$, its column minimal indices by $g_{1} \geq \cdots \geq g_{m+1-s_{1}}$, and its row minimal indices by $\tilde{r}_{1} \geq \cdots \geq \tilde{r}_{p-s_{1}}$.

By applying transposed version of Theorem 2.4 for the completion (3.11), and then Theorem 2.4 for the completion of (3.11) to (3.10), we obtain that Problem 1 is equivalent to the existence of homogeneous 
polynomials $\beta_{1}|\cdots| \beta_{n+s_{1}}$ and nonnegative integers $g_{1} \geq \cdots \geq g_{m+1-s_{1}}$ and $\tilde{r}_{1} \geq \cdots \geq \tilde{r}_{p-s_{1}}$ which satisfy

$$
\begin{aligned}
& \sum_{i=1}^{n+s_{1}} d\left(\beta_{i}\right)+\sum_{i=1}^{m+1-s_{1}} g_{i}+\sum_{i=1}^{p-s_{1}} \tilde{r}_{i}=\sum_{i=1}^{n} d\left(\alpha_{i}\right)+\sum_{i=1}^{m} c_{i}+\sum_{i=1}^{p} r_{i}+s_{1}, \\
& \beta_{i}\left|\alpha_{i}\right| \beta_{i+1}, \quad i=1, \ldots, n, \\
& 0 \leq s_{1} \leq 1, \\
& \text { if } s_{1}=0 \text { then: } \quad r_{i}=\tilde{r}_{i}, \quad i=1, \ldots, p, \\
& \qquad \mathbf{g} \prec^{\prime}\left(\mathbf{c}, b_{1}\right), \text { where } b_{1}=\sum_{i=1}^{m+1} g_{i}-\sum_{i=1}^{m} c_{i},
\end{aligned}
$$

if $s_{1}=1$, then:

$$
\begin{aligned}
& c_{i}=g_{i}, \quad i=1, \ldots, m, \\
& \mathbf{r} \prec^{\prime}\left(\tilde{\mathbf{r}}, a_{1}\right), \text { where } a_{1}=\sum_{i=1}^{p} r_{i}-\sum_{i=1}^{p-1} \tilde{r}_{i},
\end{aligned}
$$

as well as

$$
\begin{aligned}
& \sum_{i=1}^{n+s_{1}} d\left(\beta_{i}\right)+\sum_{i=1}^{m+1-s_{1}} g_{i}+\sum_{i=1}^{p-s_{1}} \tilde{r}_{i}+1-s_{1}=\sum_{i=1}^{n+1} d\left(\gamma_{i}\right)+\sum_{i=1}^{m} d_{i}+\sum_{i=1}^{p} \bar{r}_{i}, \\
& \gamma_{i}\left|\beta_{i}\right| \gamma_{i+1}, \quad i=1, \ldots, n, \\
& \text { if } 1-s_{1}=1 \text {, then: }
\end{aligned}
$$

if $1-s_{1}=0$, then:

$$
\begin{aligned}
& \bar{r}_{i}=\tilde{r}_{i}, \quad i=1, \ldots, p, \\
& \mathbf{g} \prec^{\prime}\left(\mathbf{d}, b_{1}^{\prime}\right), \text { where } b_{1}^{\prime}=\sum_{i=1}^{m+1} g_{i}-\sum_{i=1}^{m} d_{i},
\end{aligned}
$$

$$
\begin{aligned}
& d_{i}=g_{i}, \quad i=1, \ldots, m, \\
& \overline{\mathbf{r}} \prec^{\prime}\left(\tilde{\mathbf{r}}, a_{1}^{\prime}\right), \text { where } a_{1}^{\prime}=\sum_{i=1}^{p} \bar{r}_{i}-\sum_{i=1}^{p-1} \tilde{r}_{i} .
\end{aligned}
$$

Completely analogously, let us consider the pencil

$$
\left[\begin{array}{l}
A(\lambda) \\
y(\lambda)
\end{array}\right] \in \mathbb{F}[\lambda]^{(n+p+1) \times(n+m)} .
$$

Let us denote its homogeneous invariant factors by $\epsilon_{1}|\cdots| \epsilon_{n+s_{2}}$, where $s_{2} \in\{0,1\}$ is such that

$$
\operatorname{rank}\left[\begin{array}{c}
A(\lambda) \\
y(\lambda)
\end{array}\right]=n+s_{2}
$$

its column minimal indices by $e_{1} \geq \cdots \geq e_{m-s_{2}}$, and its row minimal indices by $r_{1}^{\prime} \geq \cdots \geq r_{p+1-s_{2}}^{\prime}$.

Since we have that rank of the pencil (3.10) is $n+1$, this obviously implies that we have an upper bound for $s_{1}+s_{2}$, i.e., we have

$$
0 \leq s_{1}+s_{2} \leq 1 .
$$


By applying Theorem 2.4 for the completion (3.23), and then the transposed version of Theorem 2.4 for the completion of (3.23) to (3.10), we obtain that Problem 1 is also equivalent to the existence of homogeneous polynomials $\epsilon_{1}|\cdots| \epsilon_{n+s_{2}}$ and integers $e_{1} \geq \cdots \geq e_{m-s_{2}}$ and $r_{1}^{\prime} \geq \cdots \geq r_{p+1-s_{2}}^{\prime}$ which satisfy

$$
\begin{aligned}
& \sum_{i=1}^{n+s_{2}} d\left(\epsilon_{i}\right)+\sum_{i=1}^{m-s_{2}} e_{i}+\sum_{i=1}^{p+1-s_{2}} r_{i}^{\prime}=\sum_{i=1}^{n} d\left(\alpha_{i}\right)+\sum_{i=1}^{m} c_{i}+\sum_{i=1}^{p} r_{i}+s_{2}, \\
& \epsilon_{i}\left|\alpha_{i}\right| \epsilon_{i+1}, \quad i=1, \ldots, n, \\
& 0 \leq 1 \leq s_{2}, \\
& \text { if } s_{2}=1 \text {, then: } \quad r_{i}=r_{i}^{\prime}, \quad i=1, \ldots, p, \\
& \quad \mathbf{c} \prec^{\prime}\left(\mathbf{e}, \tilde{b}_{1}\right) \text {, where } \tilde{b}_{1}=\sum_{i=1}^{m} c_{i}-\sum_{i=1}^{m-1} e_{i},
\end{aligned}
$$

if $s_{2}=0$, then:

$$
\begin{aligned}
& c_{i}=e_{i}, \quad i=1, \ldots, m, \\
& \mathbf{r}^{\prime} \prec^{\prime}\left(\mathbf{r}, \tilde{a}_{1}\right), \text { where } \tilde{a}_{1}=\sum_{i=1}^{p+1} r_{i}^{\prime}-\sum_{i=1}^{p} r_{i},
\end{aligned}
$$

as well as

$$
\begin{aligned}
& \sum_{i=1}^{n+s_{2}} d\left(\epsilon_{i}\right)+\sum_{i=1}^{m-s_{2}} e_{i}+\sum_{i=1}^{p+1-s_{2}} r_{i}^{\prime}+1-s_{2}=\sum_{i=1}^{n+1} d\left(\gamma_{i}\right)+\sum_{i=1}^{m} d_{i}+\sum_{i=1}^{p} \bar{r}_{i}, \\
& \gamma_{i}\left|\epsilon_{i}\right| \gamma_{i+1}, \quad i=1, \ldots, n+s_{2}, \\
& \text { if } 1-s_{2}=0, \text { then: } \\
& \qquad \bar{r}_{i}=r_{i}^{\prime}, \quad i=1, \ldots, p, \\
& \quad \mathbf{d} \prec^{\prime}\left(\mathbf{e}, \hat{b}_{1}\right), \text { where } \hat{b}_{1}=\sum_{i=1}^{m} d_{i}-\sum_{i=1}^{m-1} e_{i}, \\
& \text { if } 1-s_{2}=1 \text {, then: }
\end{aligned}
$$

$$
\begin{aligned}
& d_{i}=e_{i}, \quad i=1, \ldots, m, \\
& \mathbf{r}^{\prime} \prec^{\prime}\left(\overline{\mathbf{r}}, \hat{a}_{1}\right), \text { where } \hat{a}_{1}=\sum_{i=1}^{p+1} r_{i}^{\prime}-\sum_{i=1}^{p} \bar{r}_{i} .
\end{aligned}
$$

\section{Necessity:}

Let us suppose the existence of pencils $x(\lambda) \in \mathbb{F}[\lambda]^{(n+p) \times 1}, y(\lambda) \in \mathbb{F}[\lambda]^{1 \times(n+m)}$, and $z(\lambda) \in \mathbb{F}[\lambda]^{1 \times 1}$, such that the pencil

$$
\left[\begin{array}{c|c}
A(\lambda) & x(\lambda) \\
\hline y(\lambda) & z(\lambda)
\end{array}\right]
$$

is strictly equivalent to $M(\lambda)$. By Sá-Thompson's interlacing inequalities [19,20], we directly obtain $(i)$. Moreover, as we have shown above, this completion implies the existence of homogeneous polynomials $\beta_{1}|\cdots| \beta_{n+s_{1}}$ and integers $g_{1} \geq \cdots \geq g_{m+1-s_{1}}$ and $\tilde{r}_{1} \geq \cdots \geq \tilde{r}_{p-s_{1}}$ which satisfy (3.12)-(3.22), as well as the 
existence of homogeneous polynomials $\epsilon_{1}|\cdots| \epsilon_{n+s_{2}}$ and integers $e_{1} \geq \cdots \geq e_{m-s_{2}}$ and $r_{1}^{\prime} \geq \cdots \geq r_{p+1-s_{2}}^{\prime}$ which satisfy (3.25)-(3.35). Next, we have two possibilities on the value of $s_{1}$ :

- If $s_{1}=0$, then (3.15) and (3.20) give

$$
r_{i}=\bar{r}_{i}, \quad i=1, \ldots, p .
$$

Equations (3.16) and (3.21), by Theorem 2.2, imply

$$
\sum_{i=1}^{m} \min \left(c_{i}, d_{i}\right)+\max \left(c_{h}, d_{h}\right) \geq \sum_{i=1}^{m} d_{i}+b_{1}^{\prime} .
$$

Finally, since (3.18) in this case gives

$$
b_{1}^{\prime}=\sum_{i=1}^{n+1} d\left(\gamma_{i}\right)-\sum_{i=1}^{n} d\left(\beta_{i}\right)-1
$$

and since by (3.13) and (3.19) we have

$$
\operatorname{lcm}\left(\gamma_{i}, \alpha_{i-1}\right)\left|\beta_{i}\right| \operatorname{gcd}\left(\gamma_{i+1}, \alpha_{i}\right), \quad i=1, \ldots, n,
$$

we conclude

$$
\sum_{i=1}^{m} \min \left(c_{i}, d_{i}\right)+\max \left(c_{h}, d_{h}\right) \geq \sum_{i=1}^{m} d_{i}+\sum_{i=1}^{n+1} d\left(\gamma_{i}\right)-\sum_{i=1}^{n} d\left(\operatorname{gcd}\left(\alpha_{i}, \gamma_{i+1}\right)\right)-1 .
$$

Thus, if $s_{1}=0$, we have obtained $(I I)$.

- If $s_{1}=1$, then by (3.24), we have $s_{2}=0$. Hence, in this case, (3.29) and (3.34) give

$$
c_{i}=d_{i}, \quad i=1, \ldots, m,
$$

while equations (3.30) and (3.35), by Theorem 2.2, imply

$$
\sum_{i=1}^{p} \min \left(r_{i}, \bar{r}_{i}\right)+\max \left(r_{h^{\prime}}, \bar{r}_{h^{\prime}}\right) \geq \sum_{i=1}^{p} \bar{r}_{i}+\hat{a}_{1} .
$$

Finally, since (3.31) in this case gives

$$
\hat{a}_{1}=\sum_{i=1}^{n+1} d\left(\gamma_{i}\right)-\sum_{i=1}^{n} d\left(\epsilon_{i}\right)-1
$$

and since by (3.26) and (3.32) we have

$$
\operatorname{lcm}\left(\gamma_{i}, \alpha_{i-1}\right)\left|\epsilon_{i}\right| \operatorname{gcd}\left(\gamma_{i+1}, \alpha_{i}\right), \quad i=1, \ldots, n,
$$

we conclude

$$
\sum_{i=1}^{p} \min \left(r_{i}, \bar{r}_{i}\right)+\max \left(r_{h^{\prime}}, \bar{r}_{h^{\prime}}\right) \geq \sum_{i=1}^{p} \bar{r}_{i}+\sum_{i=1}^{n} d\left(\gamma_{i}\right)-\sum_{i=1}^{n} d\left(\operatorname{gcd}\left(\alpha_{i}, \gamma_{i+1}\right)\right)-1
$$


Thus, if $s_{1}=1$, we have obtained $(I)$. Altogether we have proved $(i i)$.

\section{Sufficiency:}

Let us suppose that the conditions $(i)$ and $(i i)$ are valid.

If $(I I)$ is valid, then we shall define $s_{1}:=0$, and we shall define homogeneous polynomials $\beta_{1}|\cdots| \beta_{n}$ and nonnegative integers $g_{1} \geq \cdots \geq g_{m+1}$ and $\tilde{r}_{1} \geq \cdots \geq \tilde{r}_{p}$ which satisfy (3.12), (3.13), (3.15), (3.16), (3.18), (3.19), (3.20) and (3.21).

Let

$$
\beta_{i}:=\operatorname{gcd}\left(\alpha_{i}, \gamma_{i+1}\right), \quad i=1, \ldots, n
$$

and let

$$
\tilde{r}_{i}:=r_{i}, \quad i=1, \ldots, p .
$$

Then (3.13) and (3.19) are valid. Also, since $(I I)$ holds, we have that (3.15) and (3.20) are valid as well.

Before proceeding, we note that the following is satisfied:

$$
\sum_{i=1}^{m} d_{i}+\sum_{i=1}^{n+1} d\left(\gamma_{i}\right)-\sum_{i=1}^{n} d\left(\operatorname{gcd}\left(\alpha_{i}, \gamma_{i+1}\right)\right)-1 \geq 0
$$

Indeed, we trivially have that the left hand side of (3.37) is bigger than or equal to -1 . So we only need to prove that the equality is impossible. Suppose on the contrary that

$$
\sum_{i=1}^{m} d_{i}+\sum_{i=1}^{n+1} d\left(\gamma_{i}\right)-\sum_{i=1}^{n} d\left(\operatorname{gcd}\left(\alpha_{i}, \gamma_{i+1}\right)\right)=0
$$

This implies $d_{i}=0, i=1, \ldots, m, \gamma_{1}=1$, and $\gamma_{i+1} \mid \alpha_{i}, i=1, \ldots, n$. Thus, by (3.9) and (II), we would have $\sum_{i=1}^{m} c_{i}+\sum_{i=1}^{n} d\left(\alpha_{i}\right)+1=\sum_{i=1}^{n+1} d\left(\gamma_{i}\right) \leq \sum_{i=1}^{n} d\left(\alpha_{i}\right)$, which is impossible. Hence, (3.37) holds.

Now by Theorem 2.2 condition $(I I)$ together with (3.37) implies existence of nonnegative integers $g_{1} \geq$ $\cdots \geq g_{m+1}$ such that $\mathbf{g} \prec^{\prime}(\mathbf{d}, a)$ and $\mathbf{g} \prec^{\prime}(\mathbf{c}, b)$, where

$$
a=\sum_{i=1}^{n+1} d\left(\gamma_{i}\right)-\sum_{i=1}^{n} d\left(\operatorname{gcd}\left(\alpha_{i}, \gamma_{i+1}\right)\right)-1
$$

and

$$
b=\sum_{i=1}^{m} d_{i}-\sum_{i=1}^{m} c_{i}+\sum_{i=1}^{n+1} d\left(\gamma_{i}\right)-\sum_{i=1}^{n} d\left(\operatorname{gcd}\left(\alpha_{i}, \gamma_{i+1}\right)\right)-1
$$

Hence, we have obtained (3.16) and (3.21). Also, the last with condition (3.9) gives (3.12) and (3.18), as wanted.

On the other hand, if $(I)$ is valid, then we shall define $s_{2}:=0$, and we shall define homogeneous polynomials $\epsilon_{1}|\cdots| \epsilon_{n}$ and nonnegative integers $e_{1} \geq \cdots \geq e_{m}$ and $r_{1}^{\prime} \geq \cdots \geq r_{p+1}^{\prime}$ which satisfy (3.25), (3.26), (3.29), (3.30), (3.31), (3.32), (3.34) and (3.35). 
Let

$$
\epsilon_{i}:=\operatorname{gcd}\left(\alpha_{i}, \gamma_{i+1}\right), \quad i=1, \ldots, n,
$$

and let

$$
e_{i}=c_{i}, \quad i=1, \ldots, m .
$$

Then (3.26) and (3.32) are valid. By (I), we also have (3.29) and (3.34) hold.

Also, by Theorem 2.2 condition $(I)$ together with

$$
\sum_{i=1}^{p} \bar{r}_{i}+\sum_{i=1}^{n} d\left(\alpha_{i}\right)-\sum_{i=1}^{n} d\left(\operatorname{gcd}\left(\alpha_{i}, \gamma_{i+1}\right)\right) \geq 0
$$

implies the existence of nonnegative integers $r_{1}^{\prime} \geq \cdots \geq r_{p+1}^{\prime}$ such that $\mathbf{r}^{\prime} \prec^{\prime}(\mathbf{r}, \bar{b})$ and $\mathbf{r}^{\prime} \prec^{\prime}(\overline{\mathbf{r}}, \bar{a})$, where

$$
\bar{a}=\sum_{i=1}^{n} d\left(\alpha_{i}\right)-\sum_{i=1}^{n} d\left(\operatorname{gcd}\left(\alpha_{i}, \gamma_{i+1}\right)\right)
$$

and

$$
\bar{b}=\sum_{i=1}^{p} \bar{r}_{i}-\sum_{i=1}^{p} r_{i}+\sum_{i=1}^{n} d\left(\alpha_{i}\right)-\sum_{i=1}^{n} d\left(\operatorname{gcd}\left(\alpha_{i}, \gamma_{i+1}\right)\right)
$$

Hence, we have obtained (3.30) and (3.35). Also, the last with conditions (3.9) and (I) gives (3.25) and (3.31), as wanted.

This finishes our proof.

3.2. Cases $l=0$ and $l=2$. The remaining cases $(l=0$ and $l=2)$ are covered by the results obtained in [3]. Indeed, cases $l=0$ and $l=2$ represent minimal case completion problems for the case of adding one row and one column solved in Theorems 4.2 and 4.1 in [3], respectively.

4. Conclusons. Problem 1 is motivated by larger Sá-Thompson's like problem for completion of matrix pencils by adding the same number of rows and columns, in the case when rank changes by that fixed number. This problem still remains open, but solution to Problem 1 presented in this paper (Theorem 3.1) should give us directions towards future results.

Acknowledgements. We thank the referee for suggestions that have improved the presentation of the paper.

\section{REFERENCES}

[1] I. Baragaña and A. Roca. Rank-one perturbations of matrix pencils. Preprint, arXiv:1912.08540, 2019.

[2] M. Dodig and M. Stošić. Similarity class of a matrix with prescribed submatrix. Linear Multilinear Algebra, 57:217-245, 2009.

[3] M. Dodig and M. Stošić. The general matrix pencil completion problem - a minimal case. SIAM J. Matrix Anal. Appl., 40(1):347-369, 2019.

[4] M. Dodig. Matrix pencils completion problems II. Linear Algebra Appl., 429:633-648, 2008.

[5] M. Dodig and M. Stošić. More on the properties of the generalized majorization. Preprint, arXiv:1905.08053, 2019.

[6] M. Dodig. Explicit solution of the row completion problem for matrix pencils. Linear Algebra Appl., 432:1299-1309, 2010.

[7] M. Dodig. Completion up to a matrix pencil with column minimal indices as the only nontrivial Kronecker invariants. Linear Algebra Appl., 438:3155-3173, 2013. 
[8] M. Dodig and M. Stošić. Combinatorics of column minimal indices and matrix pencil completion problems. SIAM J. Matrix Anal. Appl., 31:2318-2346, 2010.

[9] M. Dodig and M. Stošić. The rank distance problem for pairs of matrices and a completion of quasi-regular matrix pencils. Linear Algebra Appl., 457:313-347, 2014.

[10] M. Dodig. Complete characterisation of Kronecker invariants of a matrix pencil with a prescribed quasi-regular subpencil. Electron. J. Linear Algebra, 36:430-445, 2020.

[11] M. Dodig. Complete characterisation of Kronecker invariants of a quasi-regular matrix pencil with a prescribed subpencil. Preprint, 2020.

[12] M. Dodig. Matrix pencils completion problems. Linear Algebra Appl., 428(1):259-304, 2008.

[13] M. Dodig and M. Stošić. Rank one perturbations of matrix pencils. Preprint, 2019.

[14] F.R. Gantmacher. The Theory of Matrices, Vol. 2. Chelsea Publishing Company, New York, 1960.

[15] G. Hardy, J.E. Littlewood, and G. Pólya. Inequalities. Cambridge University Press, Cambridge, 1991.

[16] S. Friedland. Spectral theory of matrices I. General matrices. Mathematics Research Center, University of WisconsinMadison, 1980.

[17] S. Friedland. Matrices. Algebra, Analysis and Applications. World Scientific, New Jersey, 2016.

[18] J. Loiseau, S. Mondié, I. Zaballa, and P. Zagalak. Assigning the Kronecker invariants of a matrix pencil by row or column completion. Linear Algebra Appl., 278:327-336, 1998.

[19] E.M. de Sá. Imbedding conditions for $\lambda$-matrices. Linear Algebra Appl., 24:33-50, 1979.

[20] R.C. Thompson. Interlacing inequalities for invariant factors. Linear Algebra Appl., 24:1-31, 1979.

[21] I. Zaballa. Matrices with prescribed rows and invariant factors. Linear Algebra Appl., 87:113-146, 1987. 